\title{
A RECENTLY DEVELOPED BIFACIAL PLATELET-RICH FIBRIN MATRIX
}

\author{
E. Lucarelli ${ }^{*}$, R. Beretta ${ }^{2}$, B. Dozza ${ }^{1}$, P.L. Tazzari ${ }^{3}$, S.M. O’Connell ${ }^{4}$, F. Ricci ${ }^{3}$, M. Pierini ${ }^{1}$,
} S. Squarzoni ${ }^{5}$, P.P. Pagliaro ${ }^{3}$, E.I. Oprita ${ }^{6}$, and D. Donati ${ }^{1}$

\author{
${ }^{1}$ Bone Regeneration Laboratory, Dipartimento di Patologie Ortopediche-Traumatologiche Specialistiche, Rizzoli \\ Orthopaedic Institute, Bologna, Italy \\ ${ }^{2}$ Cascade Medical Enterprises LLC, Research and Development, Wayne NJ, USA \\ ${ }^{3}$ Service of Transfusion Medicine, S. Orsola-Malpighi Hospital, Bologna, Italy \\ ${ }^{4}$ Department of Vascular and General Surgery, Englewood Hospital \& Medical Center, Mt. Sinai School of \\ Medicine, Englewood, NJ, USA \\ ${ }^{5}$ Institute of Molecular Genetics (IGM)-CNR, Unit of Bologna c/o Rizzoli Orthopaedic Institute, Bologna, Italy \\ ${ }^{6}$ National Institute R\&D for Biological Sciences, Bucharest Romania
}

\section{Abstract}

Platelet-rich plasma (PRP) is used clinically in liquid or gel form to promote tissue repair. Because of the poor mechanical properties, conventional PRP is often difficult to handle when used in clinical settings and requires secure implantation in a specific site, otherwise when released growth factors could be washed out during an operation. In this study, we analyzed the end product of a recently developed commercially available system (FIBRINET ${ }^{\circledR}$ ), which is a dense pliable, platelet-rich fibrin matrix (PRFM). We characterized the mechanical properties of PRFM and tested whether PRFM releases growth factors and whether released factors induce the proliferation of mesenchymal stem cells (MSC). Mechanical properties as well as platelet distribution were evaluated in PRFM. PRFM demonstrated robust mechanical properties, with a tear elastic modulus of $937.3 \pm 314.6 \mathrm{kPa}$, stress at a break of $1476.0 \pm 526.3$ $\mathrm{kPa}$, and an elongation at break of $146.3 \pm 33.8 \%$. PRFM maintained its mechanical properties throughout the testing process. Microscopic observations showed that the platelets were localized on one side of the matrix. Elevated levels of PDGF-AA, PDGF-AB, EGF, VEGF, bFGF and TGF- $\beta 1$ were measured in the day 1-conditioned media $(\mathrm{CM})$ of PRFM and growth factor levels decreased thereafter. BMP2 and BMP7 were not detectable. MSC culture media supplemented with 20\% PRFM-CM stimulated MSC cell proliferation; at 24 and 48 hours the induction of the proliferation was significantly greater than the induction obtained with media supplemented with $20 \%$ foetal bovine serum. The present study shows that the production of a dense, physically robust PRFM made through high-speed centrifugation of intact platelets and fibrin in the absence of exogenous thrombin yields a potential tool for accelerating tissue repair.

Keywords: Platelet-rich fibrin matrix (PRFM), platelet-rich plasma (PRP), physical properties, fibrin, mesenchymal stem cells.

*Address for correspondence:

Enrico Lucarelli

Bone Regeneration Laboratory,

Dipartimento di Patologie Ortopediche-Traumatologiche Specialistiche, Rizzoli Orthopaedic Institute,

Via di Barbiano 1/10, I-40136, Bologna, Italy

Telephone Number: +390516366595

FAX Number: +390516366799

E-mail: enrico.lucarelli@ior.it
Platelets are known to release a variety of factors on activation. These factors have a positive effect on tissue repair. Platelets are easily collected from the blood stream and are concentrated in a small volume of plasma known as platelet-rich plasma (PRP). PRP has been used in a variety of surgical settings for soft-tissue healing enhancement, including chronic lower extremity ulcer healing (Mazzucco et al., 2004; O'Connell et al., 2008), and tendon healing (Franchini et al., 2006; Gamradt et al., 2007; Maniscalco et al., 2008; Sánchez et al., 2007; Virchenko and Aspenberg, 2006). It is also used in orthopaedic and oral maxillofacial surgery where it accelerates autogenous bone graft healing (Fennis et al., 2004; Marx et al., 1998; Simon et al., 2009), and in cosmetic surgery (Man et al., 2001). PRP has also been used in combination with mesenchymal stem cells (MSC) to promote bone regeneration (Hibi et al., 2006; Ito et al., 2006; Kitoh et al., 2004; Kitoh et al., 2007; Lucarelli et al., 2003; Pieri et al., 2008) or mixed with fat cells for breast reconstruction, to correct painful, adherent scars, and to solve progressive hemifacial atrophy (Azzena et al., 2007; Cervelli et al., 2009a; Cervelli et al., 2009b; Cervelli et al., 2009c; Cervelli et al., 2009d; Fulton, 2003).

Once injected or implanted, PRP is thought to release growth factors locally for several days, inducing accelerated tissue repair (O'Connell et al., 2006). Currently, several methods and systems are available for the preparation of PRP, most producing a liquid end product. The physical properties of PRP can be changed if plasma and platelets are stimulated, usually by the addition of $\mathrm{CaCl}_{2}$ and bovine thrombin, to produce a fibrin network. In this case, the end product is generally a weak gel. The physical properties of fibrin gels have been elucidated in previous studies. These studies have demonstrated that the use of different thrombin-fibrinogen concentration ratios greatly affected the gel structure (Blomback and Okada, 1982; Blomback et al., 1989). Other factors such as protein and ion concentrations (Chung and Shu, 1990; Okada et al., 1983; Okada et al., 1985), including calcium, also have an effect on gel structure (Okada and Blomback, 1983). Among the commercially available systems, a recently developed system has been developed (FIBRINET ${ }^{\circledR}$ ) in which the end product, called platelet-rich fibrin matrix (PRFM), is produced with the combined use of gravitational force and calcium without the use of exogenous thrombin and appears physically stronger than conventional PRPs. In 
this study, we characterized the mechanical properties of PRFM and tested whether growth factors released from PRFM could support MSC proliferation, making it suitable for a variety of tissue engineering applications.

\section{Materials and Methods}

\section{PRFM Preparation}

PRFM was prepared according to the manufacturer's instructions (FIBRINET ${ }^{\circledR}$, Cascade Medical Enterprises, Wayne, NJ, USA). Briefly, $18 \mathrm{~mL}$ of human blood were collected into sterile supplied blood collection/separation tubes from healthy consenting blood donors $(n=5)$. After gentle mixing, the tubes were centrifuged at $1100 \mathrm{~g}$ for 6 minutes to obtain PRP. PRP was transferred into a calcium chloride-containing glass $\left(0.25 \mathrm{~mL} \mathrm{CaCl}_{2} 1 \mathrm{M}\right)$ bottle under sterile conditions. The bottle was gently swirled and then immediately placed in a swing-out rotor centrifuge (Heraeus $\mathrm{GmbH}$, Hanau, Germany) and centrifuged at $4500 \mathrm{~g}$ for 25 minutes at $25^{\circ} \mathrm{C}$. A translucent, yellow-white platelet-fibrin matrix, having the same inner diameter as the bottle $(33 \mathrm{~mm})$ was recovered at the bottom. All of these matrices were tested for tensile strength and elastic modulus five days after preparation.

\section{Blood Parameter Measurements}

After informed consent venous blood was obtained from healthy donors, platelet counts were performed on the donors' whole blood and on the residual serum remaining after PRFM preparation with a Coulter Haematology Analyzer (Beckman Coulter Inc., Brea, CA, USA). Fibrinogen concentration was measured with the Stago Coagulation System employing the Clauss method (Roche, Basel, Switzerland) on the same samples. For PRP, the standard method was used (calibration for a linearity range from 100 to $600 \mathrm{mg} / \mathrm{dL}$ ), while for serum, the ultrasensitive method was selected (calibration for a linearity range from 1 to $50 \mathrm{mg} / \mathrm{dL}$ ). These analyses were performed on different samples with respect to the ones used for the mechanical tests.

\section{Characterization of the PRFM}

Immunofluorescence Confocal Microscopy. PRFM were cut into 5-mm squares for labelling with fluorescent antibody $(\mathrm{Ab})$ in order to investigate the tridimensional microscopic structure of the matrix using laser confocal microscopy. An indirect labelling procedure was used to fluorescently stain the membrane fibrin. The samples were washed twice with a $10 \%$ glycine solution followed by two more washes with $150 \mathrm{mM}$ phosphate buffered saline (PBS) containing $1 \%$ bovine serum albumin (BSA). The samples were then incubated overnight with a solution of the primary antibody, Goat $\mathrm{F}(\mathrm{ab})_{2}$, fragment specific to human fibrinogen (ICN, Eschwege, Germany). The next day, the samples were washed for 1 hour in $20 \mathrm{mM}$ PBS and $0.3 \%$ Triton X-100 and then treated with the secondary antibody (Donkey anti-goat Ab) labelled with Texas Red (Jencons, Bedfordshire, UK). The samples were then washed thoroughly with $20 \mathrm{mM}$ PBS and $0.3 \%$ Triton X100 and mounted with $90 \%$ glycerol on glass slides for microscopic observation. PRFM organization was assessed using the Bio-Rad MRC-1024 confocal microscope (BioRad Laboratories, Hercules, CA, USA) equipped with a krypton/argon laser. Incident wavelength was $568 \mathrm{~nm}$ for Texas Red. Microphotographs were taken using a Focus Imagecorder Plus (Focus Graphic Inc., St. Louis, MO, USA) on Kodak Film.

Scanning Electron Microscopy. Membrane samples were cut into uniform shapes, washed with $0.1 \mathrm{M}$ cacodylate buffer (pH 7.3) and fixed with 2.5\% glutaraldehyde in 0.1 $\mathrm{M}$ cacodylate buffer $(\mathrm{pH} 7.3)$ for 1 hour at $4^{\circ} \mathrm{C}$. After a second wash with $0.1 \mathrm{M}$ cacodylate buffer $(\mathrm{pH} 7.3)$, the samples were post-fixed with $1 \%$ osmium tetroxide $(\mathrm{OsO} 4)$ $-0.1 \mathrm{M}$ cacodylate buffer for 1 hour at room temperature $\left(22 \pm 2^{\circ} \mathrm{C}\right)$. After rinsing with $0.1 \mathrm{M}$ cacodylate buffer, the samples were dehydrated in an ethanol series and critical point dried. The samples were then mounted on aluminium stubs with silver adhesive paint, sputter-coated with $4 \mathrm{~nm}$ gold in an argon atmosphere in an Edwards (Crawley, West Sussex, U.K.) S 150 B apparatus, and observed at $0^{\circ}$ tilt angle with a Cambridge (Cambridge, UK) Stereoscan 200 scanning electron microscope operated at $20 \mathrm{kV}$. For scanning electron microscopy, two matrices were obtained from each of $18 \mathrm{~mL}$ of blood.

Morphological Analysis. Average fibre diameter was measured on digitized micrographs obtained by scanning electron microscopy experiments. Each measure was taken on 150 fibres on both sides of each membrane. Subsequently, mean values and standard deviation (SD) were calculated.

Mechanical Properties. To study the mechanical properties of PRFM, specimens were fabricated by cutting out from each circular PRFM membrane a test specimen in accordance to ASTMD 1708. All of these specimens were stored in sterile saline solution at $4^{\circ} \mathrm{C}$ and tested after five days from preparation. All tests were performed at $25^{\circ} \mathrm{C}$. Tensile elastic modulus, elongation at break, and tensile strength (peak breaking energy) were evaluated according to ASTM D 882:2209 and ASTM D 1708 standard procedures respectively, using an Instron (Norwood, MA, USA) 4302 mechanical testing station with a loading cell of $10 \mathrm{~N}$. Particular attention was paid to fix the sample on the holder of the testing station. We used elastomeric tags to achieve secure, uniform grip of every sample and to avoid possible damage to the strips.

\section{PRFM Conditioned Media Collection}

Immediately following their preparation, PRFM prepared from 3 different donors were placed in a 6-well plate where they were submerged in $3 \mathrm{ml}$ of serum-free culture media a-modified minimum essential medium ( $\alpha$-MEM, Sigma Chemical, St. Louis, USA) and incubated in a humidified atmosphere at $37^{\circ} \mathrm{C}$ with $5 \%$ carbon dioxide. PRFMconditioned media (CM) containing the eluted growth factors were collected in their entirety and an equal volume of fresh serum-free $\alpha$-MEM was added back into each well at 1, 2, 3 and 7 days. All collected CM samples were divided into aliquots and immediately stored at $-80^{\circ} \mathrm{C}$ until 
testing. CM were utilized to quantify growth factor concentrations and to investigate their ability to promote MSC proliferation.

\section{Quantification of growth factors released from PRFM}

$\mathrm{CM}$ collected from all time points were assayed for plateletderived growth factor (PDGF)-AA, platelet-derived growth factor (PDGF)-AB, transforming growth factor (TGF) $\beta$ 1 , vascular endothelial growth factor (VEGF), epidermal growth factor (EGF), basic fibroblast growth factor (bFGF), bone morphogenetic protein (BMP)-2, and bone morphogenetic protein (BMP)-7. The growth factor concentrations were assayed by quantitative enzyme-linked immunosorbent assays (ELISA) (all human Quantikine ${ }^{\mathbb{R}}$ ELISA kits were obtained from R\&D Systems (Minneapolis, MN, USA) according to manufacturer's instructions. Briefly, the samples and standards were prepared in duplicate according to the manufacturer's protocol and added to 96 growth factor antibody coated well plates. The plates were incubated at room temperature, washed, and incubated with selective enzyme-conjugated antibodies for an additional time at room temperature. The wells were then washed and substrate was added for 30 minutes at room temperature. Stop solution was added to each well, and the absorptions at $450 \mathrm{~nm}$ were determined using a microplate photometer (Synergy HT, BioTek Instruments Inc., Winooski, VT, USA). Standard curves for each growth factor were generated and growth factor concentrations $(\mathrm{pg} / \mathrm{mL})$ of each sample were determined. Samples were measured in appropriate dilutions prepared as required to fit the respective calibration curves. The minimum detection limits of these assay reported by the manufacturer are $2.3 \mathrm{pg} / \mathrm{mL}$ for PDGF-AA, $1.7 \mathrm{pg} / \mathrm{mL}$ for PDGF-AB, $4.6 \mathrm{pg} / \mathrm{mL}$ for TGF $\beta 1,5.0 \mathrm{pg} / \mathrm{mL}$ for VEGF, $0.7 \mathrm{pg} / \mathrm{mL}$ for EGF, $3.0 \mathrm{pg} / \mathrm{mL}$ for $\mathrm{bFGF}, 11 \mathrm{pg} / \mathrm{mL}$ for BMP-2 e $2.4 \mathrm{pg} / \mathrm{mL}$ for BMP-7.

\section{Mesenchymal stem cell isolation, culture and growth kinetic determination}

Growth factors released from platelets have been shown to promote MSC proliferation (Doucet et al., 2005; Lucarelli et al., 2003). The ability of factors released from PRFM to support proliferation of primary MSC cultures was tested on MSC isolated from 3 patients undergoing elective surgery at Rizzoli Orthopaedic Institute. Cultures were established after obtaining informed consent and according to a protocol approved by the Rizzoli's Ethics Committee. Briefly, a $10 \mathrm{~mL}$ bone marrow sample was aspirated from the anterior iliac crest. Mononuclear cells were isolated in a density gradient and suspended in $(\alpha-$ MEM) containing 20\% foetal bovine serum (FBS, Euroclone, Wetherby, UK). All the nucleated cells were plated in $150 \mathrm{~cm}^{2}$ culture flasks and incubated in a humidified atmosphere at $37^{\circ} \mathrm{C}$ with $5 \%$ carbon dioxide. Non-adherent cells were discarded after 3 days, and adherent cells were cultured for further expansion. When cultured flasks reached near confluence, cells were detached by mild trypsinization and reseeded onto new flasks at one-third density for continued passage. Media were changed every 3 to 4 days.
Passage 3 MSC were seeded in 96-well plates (tissue culture grade, flat bottom) in serum-free $\alpha$-MEM at an initial density of $5 \times 10^{3}$ cells/well (that is, approximately $40 \%$ confluence). After overnight attachment, serum-free medium was removed and cells were cultured in $\alpha$-MEM supplemented $5 \%, 10 \%$ or $20 \%$ day 1 PRFM-CM. MSC maintained in normal cell culture medium $(\alpha$-MEM supplemented with $20 \%$ FBS) or serum free medium $(\alpha-$ MEM) were used as positive and negative controls, respectively. Cell proliferation was assessed after 24, 48, and 72 hours by methylene blue assay (Oliver et al., 1989). Briefly, cells were fixed by adding $100 \mu \mathrm{l}$ of $10 \%$ formol saline to each well for 30 minutes. Cells were then stained with $100 \mu \mathrm{l}$ of filtered $1 \%(\mathrm{w} / \mathrm{v})$ methylene blue in $0.01 \mathrm{M}$ borate buffer ( $\mathrm{pH} 8.5$ ) added to each well. After 30 minutes, excess dye was removed. The remaining dye was then

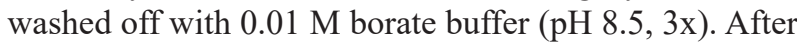
the last rinse the cell layer still stained with methylene blue was examined microscopically. The dye was then eluted by adding $100 \mu \mathrm{l}$ of $1: 1(\mathrm{v} / \mathrm{v})$ ethanol and $0.1 \mathrm{M}$ $\mathrm{HCl}$ to each well. The plates were then gently shaken and the absorbance at $655 \mathrm{~nm}\left(\mathrm{~A}_{655}\right)$ measured for each well by a microplate photometer (Synergy HT, BioTek Instruments Inc.). The photometer was blanked on control wells containing elution solvent alone. Each treatment condition was performed in quadruplicate. A linear relationship between absorbance, determined in the assay, and cell number was verified by counting the cells with a NucleoCounter (Chemometec, Allerod, Denmark).

\section{Statistical Analysis}

Results were determined from a minimum of three independent experimental data sets with duplicate measurements per experiment. Data are expressed as mean \pm standard deviation. Statistical analysis was performed by one-way analysis of variance (ANOVA). When significance was detected, post-hoc comparisons between different groups were made using Bonferroni's test for multiple comparisons. A probability of less than 0.05 was considered to be statistically significant. The statistical program GraphPad - Prism4 (GraphPad Software, San Diego, CA, USA) was used for the analysis.

\section{Results}

\section{PRFM Description}

To investigate the efficiency of the preparation procedure, we evaluated fibrinogen levels before and after matrix formation. Results shown in Table 1 demonstrate that no detectable fibrinogen was left in the residual serum. These results indicate that all the fibrinogen originally present within the PRP was polymerized to fibrin in the PRFM. In addition, blood parametric analysis of the residual serum showed that only $0.9 \%$ of platelets were left in the serum, indicating that $99.1 \%$ of the platelets were present in the PRFM (Table 1). Platelet recovery data from PRP obtained with the PRFM FIBRINET ${ }^{\circledR}$ kit have been previously published from Leitner et al. being $65.5 \%$ (Leitner et al., 2006; Mazzucco et al., 2009). Considering the platelet concentration factor represented by the PRFM, use of the 
Table 1. Fibrinogen and Platelet Concentration in PRP, Whole Blood, and Residual Serum after Platelet-Rich Fibrin Matrix Preparation (Residual Serum). Data are presented as mean \pm standard deviation $(n=5)$.

\begin{tabular}{|c|c|}
\hline & Fibrinogen concentration (mg/dL) \\
\hline PRP & $325.20 \pm 47.42$ \\
\hline Residual Serum & Undetectable \\
\hline & Platelet concentration (platelet number/mm $\mathbf{m m}^{3}$ \\
\hline Whole Blood & $299,000 \pm 80,346$ \\
\hline Residual Serum & $2,600 \pm 547.72 * * *$ \\
$*$
\end{tabular}

PRFM kit produces a 210-fold higher concentration of platelets and fibrin when compared to the initial input whole blood volume.

Macroscopically, the PRFM is a translucent yellowwhite disk (Fig. 1A) of $0.105 \pm 0.021 \mathrm{~mm}$ thickness and $33 \mathrm{~mm}$ diameter, with both sides of the membrane appearing the same. PRFM is easy to handle and does not tear when manipulated with forceps (Fig. 1B and 1C). We observed that the PRFM produced by the FIBRINET ${ }^{\circledR}$ kit appeared stable. Confocal microscopy was used to observe fibrin architecture in PRFM labelled with a fluorescent antibody against human fibrinogen (Fig. 2). PRFM consisted of a very compact, coarse, fibrin network. Scanning electron micrographs of the PRFM revealed that the two sides of the matrix are different. On one side, platelets are visible within the fibrin network (Fig. 3A and $3 \mathrm{C}$, see Fig. 3D and $3 \mathrm{~F}$ for higher magnification). In particular there was a region (Fig. $3 \mathrm{C}$ and $3 \mathrm{~F}$ ) in which platelets and cells completely covered the fibrin network. Platelets observed have mostly a lenticular shape that is consistent with an inactivated state. The larger cells observed are consistent with residual white blood cells. On the reverse side of the matrix no platelets or cells were visible, only the fibrin network (Figs. 3B and 3E). The morphological analysis of the fibrin network shows that, on the upper side of the membrane, the fibres are organized in twisted parallel strands and bundles, frequently reaching considerable diameters up to $1.1 \mu \mathrm{m}$. The average diameter of isolated fibres in this side is close to what would be expected for a native fibrin clot, dehydrated and critical point-dried and analyzed with scanning electron microscopy (Collet et al., 2004; Collet et al., 2005). The average diameter measured on this side of PRFM is 63.6 $\pm 30.3 \mathrm{~nm}$.
Table 2. Mechanical Characteristics of PRFM. Tensile strength (stress and elongation at break) and elastic modulus were measured. Data are expressed as mean \pm standard deviation of three determination of five donors.

\begin{tabular}{|c|c|c|}
\hline \multicolumn{3}{|c|}{ PRFM Tensile Properties } \\
\hline $\begin{array}{c}\text { Stress at } \\
\text { break (KPa) }\end{array}$ & $\begin{array}{c}\text { Elongation at } \\
\text { Break (\%) }\end{array}$ & $\begin{array}{c}\text { Elastic } \\
\text { Modulus (kPa) }\end{array}$ \\
\hline $1476 \pm 526.3$ & $146.3 \pm 33.8$ & $937.3 \pm 314.6$ \\
\hline
\end{tabular}

On the bottom of the membrane, where fibres are pressed against the flat surface of the glass container and subjected to high centrifugal force stress, we can clearly see two distinct populations: one showing sizes that are close to the native fibres with an average diameter of 95.7 $\pm 29.5 \mathrm{~nm}$; the second population has an average diameter of $305.3 \pm 95.9 \mathrm{~nm}$. This second population consists mainly of "pressed" bundles made of smaller fibres packed longitudinally. Both populations are short fibres and bundles that are linked through a high density branch. On this lower side of the membrane, the formation of large cables made of fused parallel fibres and bundles is even more pronounced. Large areas of the underside of the membrane consist of fibre constructs that are strongly pressed against the glass surface of the container forming dense aggregates, with no pores. The microscopic view shows an alternating pattern of large strands and bundles with obvious pores, and thick, non-porous areas, consisting of fused strands and amorphous fibrin (Fig. 3D). There is also evidence of fibres and bundles with a kinked structure. In general, both sides of the membrane demonstrated very high fibre density compared to native fibrin clots.
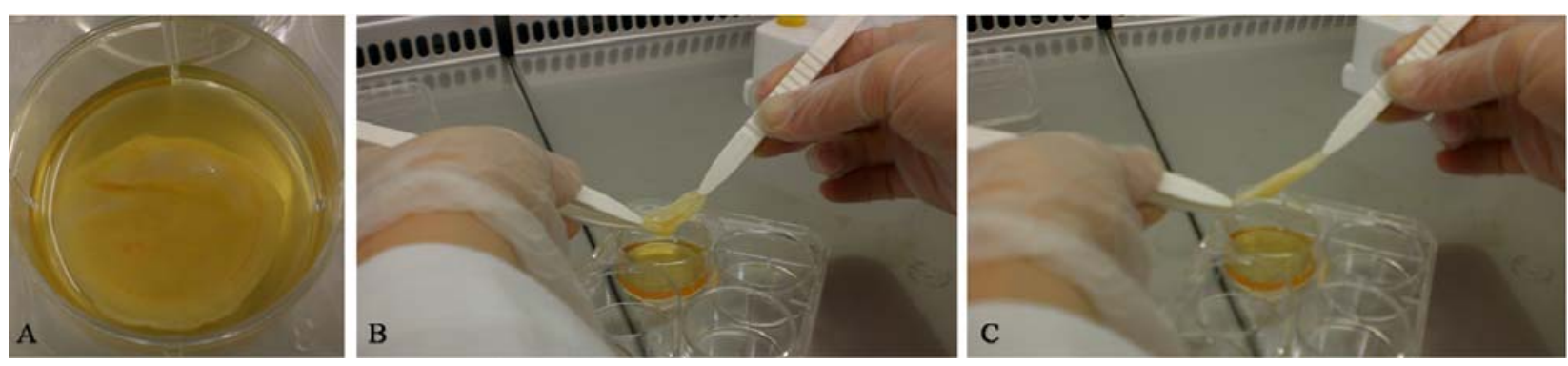

Fig. 1. (A) Macroscopic image of the PRFM. (B) PRFM before, and (C) after it is subjected to manual strain with a forceps. 


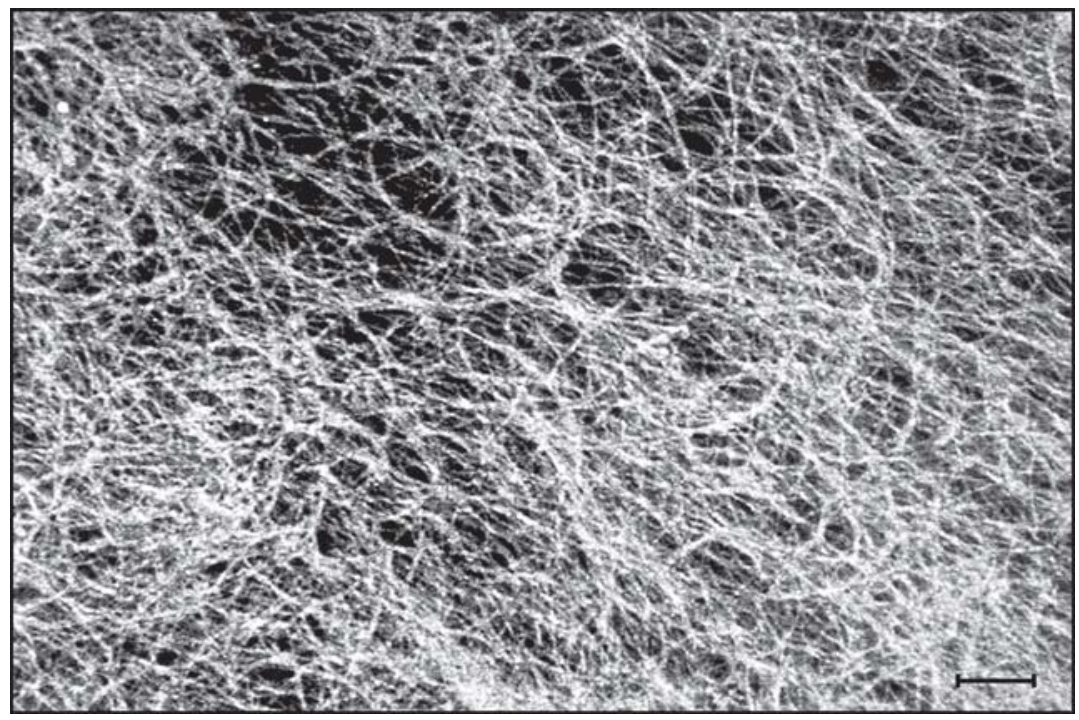

Fig. 2. Confocal fluorescence microscopic image of fibrin fibrils in a PRFM highlighted by incorporation of fluorescent-labelled indirect antifibrinogen antibody system. The picture was taken in a representative field. The scale bar $=10 \mu \mathrm{m}$.

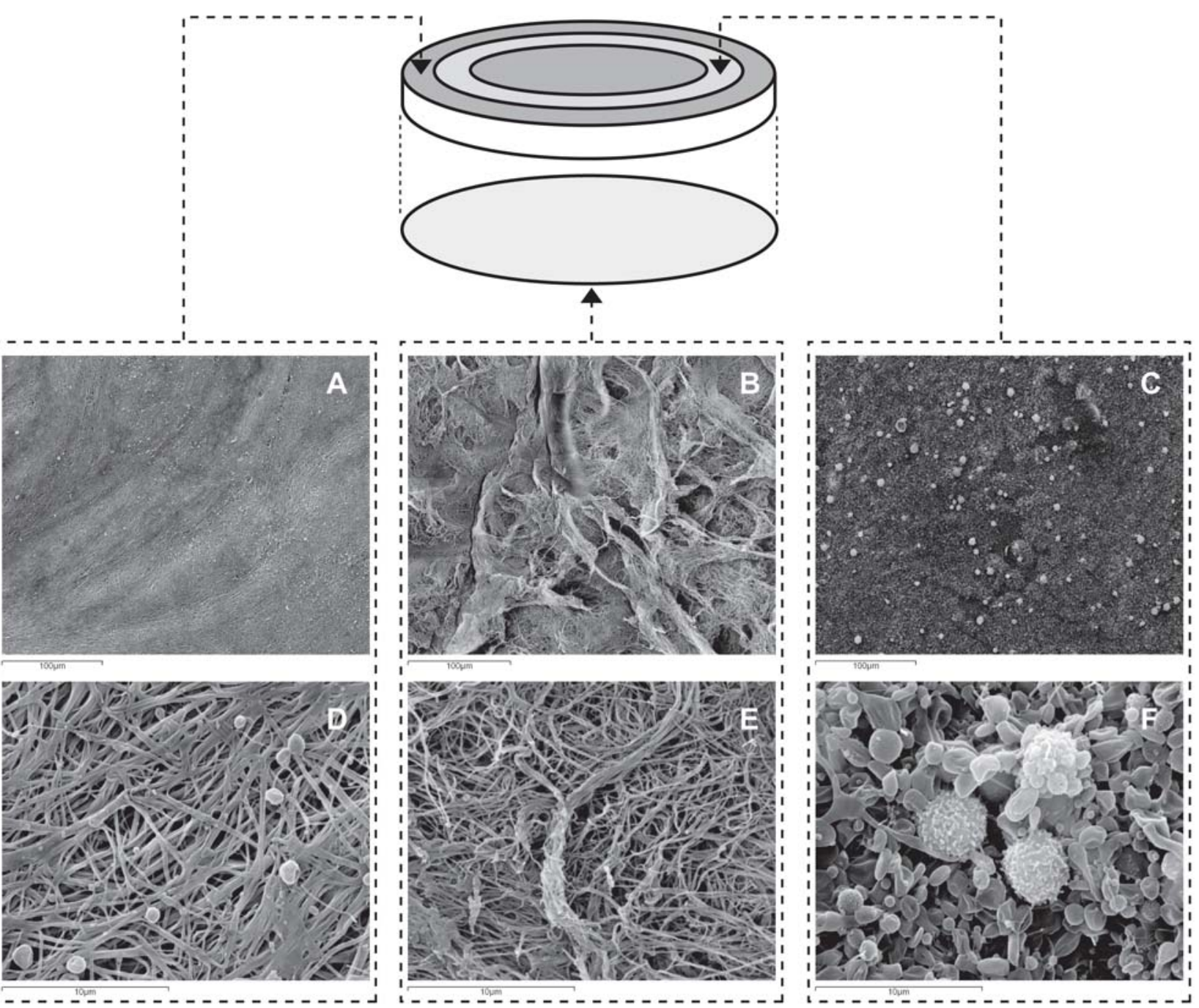

Fig. 3. Scanning electron micrograph images of the PRFM. (A) Representative picture obtained at the periphery of the disk. (B) The reverse side of the membrane in which no platelets or cells are visible, but a coarse surface of fibrin strands and bundles is visible. (C) Representative picture of the platelet/cell-rich area. (D) The same field as in A at higher magnification shows platelets on the surface of the fibrin network. (E) A higher magnification of the same field as in B of the reverse side of the membrane in which no platelets or cells are visible, but a coarse surface of fibrin strands and bundles is visible. (F) A higher magnification of the same field as in $\mathrm{C}$ shows a thick layer of unactivated platelets in which a few nucleated cells are visible. 
A

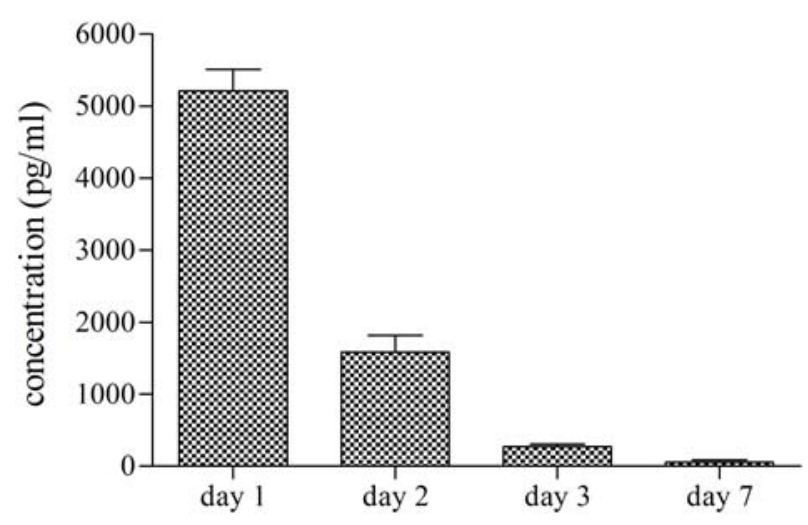

C

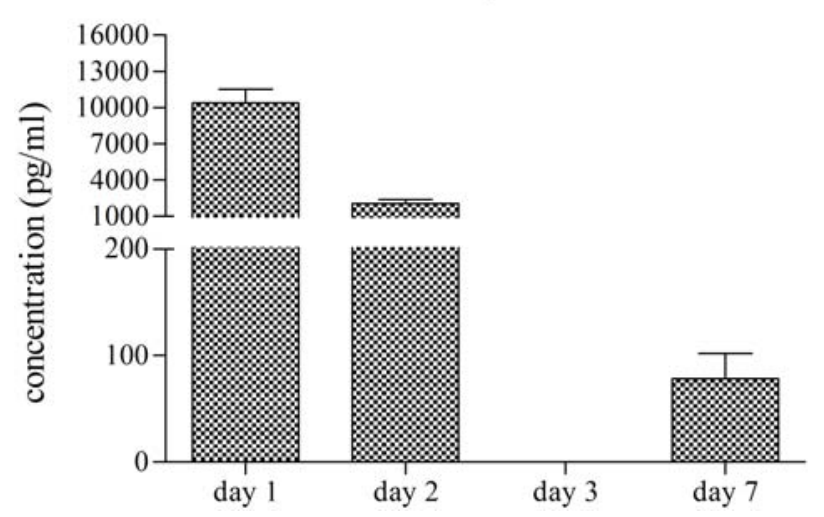

E

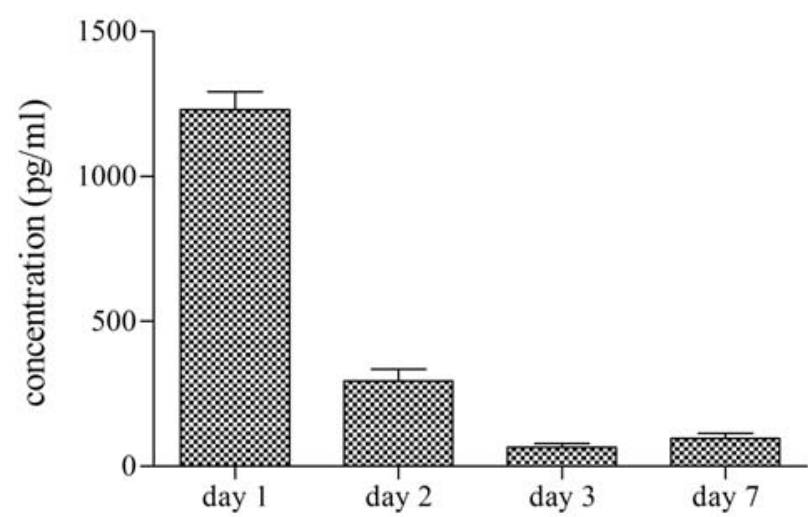

B

PDGF-AB

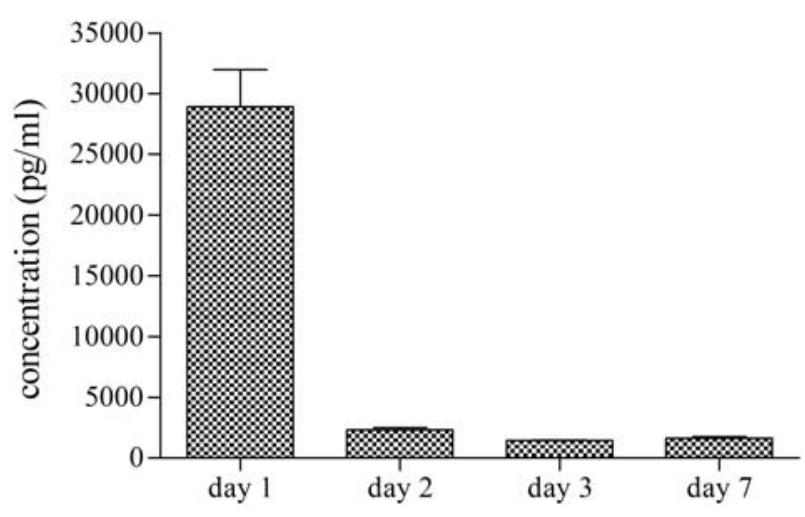

D

VEGF

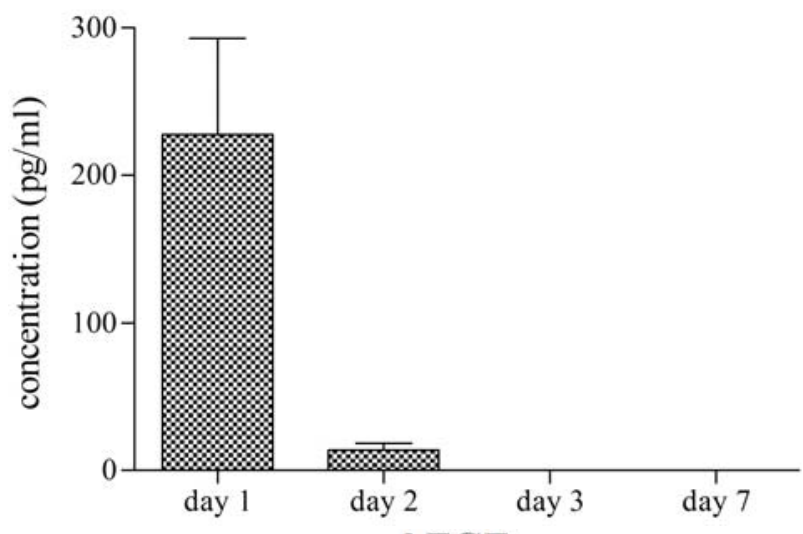

bFGF

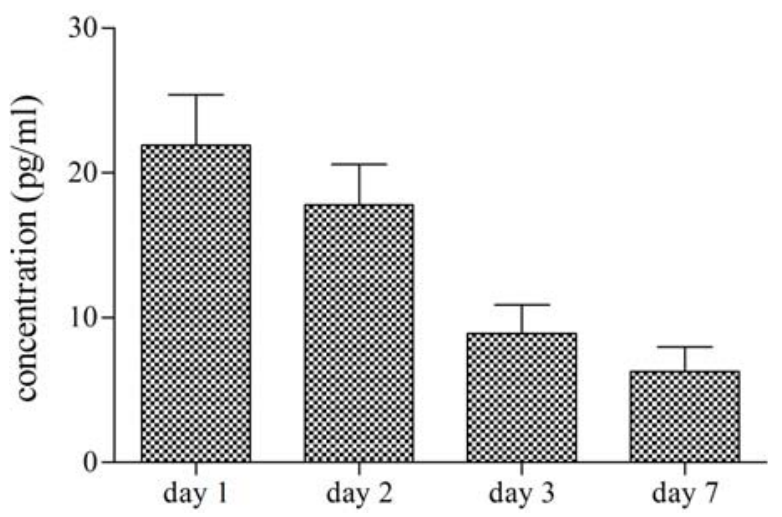

Fig. 4. Quantification of growth factor release in the PRFM- conditioned media (CM) obtained from three donors. Mean concentrations $\pm \mathrm{SD}(\mathrm{n}=3)$ of the indicated growth factors in the PRFM-CM at the indicated time point of CM collection are presented. (A) PDGF AA, (B) PDGF-AB, (C) TGF- $\beta 1$, (D) VEGF, (E) EGF and (F) bFGF.

\section{Mechanical Properties}

Mechanical testing showed that PRFM has enhanced mechanical properties with respect to all the other platelet gels described in the literature, with a tear elastic modulus (expression of the stiffness) of $937.3 \pm 314.6 \mathrm{kPa}$ and stress at break of $1476.0 \pm 526.3 \mathrm{kPa}$, while the elongation at break reaches $146.3 \% \pm 33.8 \mathrm{kPa}$ (Table 2). Mechanical properties of samples kept refrigerated in a saline solution for 18 days were not significantly different compared to the ones measured after 5 days from preparation (data not shown).

\section{Growth factors released from PRFM}

The concentration of PDGF-AA, PDGF-AB, TGF- $\beta 1$, VEGF, EGF and bFGF in the PRFM-CM was greater at day 1 compared to day 2, day 3 and day 7 (Fig. 4). These results are consistent with an abundant release of the growth factors within the first day, and a gradual decrease of the release of the growth factors thereafter. The kinetics of release varied among growth factors, release was greatest within the first day for VEGF (Fig. 4D), while a more gradual release was seen with bFGF (Fig. 4F). BMP2 and BMP-7 were always below the level of detection.

\section{MSC proliferation induced by PRFM-CM}

The results of the methylene blue assay performed on the MSC obtained from three different donors subjected to different concentrations of day 1-PRFM-CM are shown in Fig. 5. The increase in MSC proliferation of 5\%,10\% and $20 \%$ day 1 -PRFM-CM was tested up to 72 hours. 


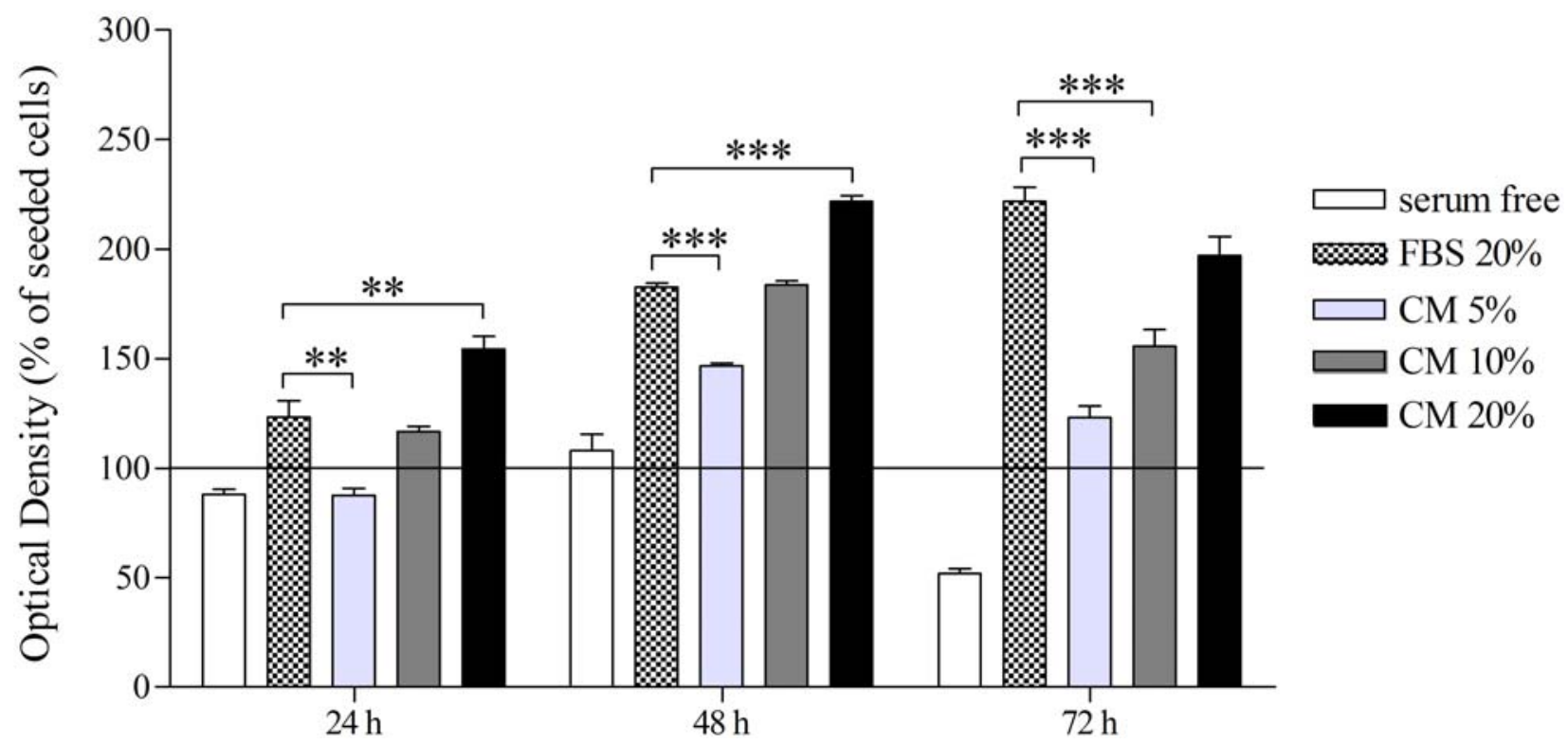

Fig. 5. PRFM-conditioned media (CM) induced cell proliferation. MSC proliferation was determined by methylene blue assay after 24, 48, and 72 hours in culture. Mean optical density values displayed as \% of seeded cells referred as $100 \%$ (line). Data represent mean values \pm SD of measurements obtained from MSC harvested from 3 donors. CM were obtained from PRFM prepared from 3 donors. Each sample was assayed in quadruplicate. ${ }^{* *} p<0.01, * * *$ $p<0.001$. Statistical significance was not reached $(p>0.05)$ where $*$ for comparisons are not shown.

Optical density values indicated relative cell proliferation. Compared to the serum-free control, a statistically significant difference was observed at all time points and with all concentrations of PRFM-CM tested (Fig. 5). These data indicate that factors released from the PRFM are active and sustain the proliferative potential of MSC. The extent of proliferation of MSC cultured with medium supplemented with 5\% day 1-PRFM-CM up to 72 hours was significantly lower from the proliferation observed in MSC supplemented with 20\% FBS. Medium supplemented with $10 \%$ of day 1 -PRFM-CM support MSC proliferation as $20 \%$ FBS up to 48 hours, while the effect significantly decreased after 72 hours. MSC proliferation was significantly increased by $20 \%$ day 1 -PRFM-CM compared to $20 \%$ FBS up to 48 hours, while the effects of the two supplemented media were not significantly different after 72 hours.

\section{Discussion}

Conventional PRP is usually produced during a two-step procedure (Fennis et al., 2004; Marx et al., 1998; Mazzucco et al., 2004; Okada et al., 1983; Sánchez et al., 2007). In the first step, PRP is formed by separation of a platelet concentrate from the platelet poor plasma and the white and red cell fraction. In the second step, exogenous thrombin, or other activator such as bathroxobin (Mazzucco et al., 2008) is added together with calcium chloride, or calcium gluconate, to the platelet concentrate and the platelet poor plasma. This converts fibrinogen into fibrin, and the fibrin network is formed. In contrast, the PRFM system isolates plasma and platelets in the first centrifugation using the thixotropic separator gel in the first tube. In the second step, PRP is re-calcified in the absence of exogenous thrombin and centrifuged for the second time at high gravitational force to form the PRFM.

PRFM prepared with FIBRINET ${ }^{\circledR}$ system has been used previously to treat difficult lower-extremity ulcers as described by O'Connell and co-workers (O'Connell et al., 2006; O'Connell et al., 2008). Possible reasons for the success of PRFM in closing these difficult ulcers may be due to its mechanical properties, its localized high platelet concentration and its elevated cytocompatibility as a result of its being produced directly from the patient's own blood without any exogenous organic additives. The results of the studies described here show that the mechanical properties of an autologous platelet-fibrin preparation can be improved by inducing extensive fibrin network formation through increased gravitational force in the second centrifugation during PRFM preparation. With this procedure, PRP can be produced in the form of a PRFM. Conventional PRPs are usually liquids or weak gels, and fibrin-based clots present an elastic modulus, or stiffness, measured in the range of 0.1 to $1500 \mathrm{~Pa}$, depending on the clot structure and the physiologic conditions (Bale et al., 1985; Carr et al., 1995; Collet et al., 2004; Collet et al., 2005; Cummings et al., 2004; Urech et al., 2005; Weisel, 2004). In contrast, PRFM made of the same components in our experiments, gives an elastic modulus of $937.3 \mathrm{kPa}$. This level is comparable with that of arterial tissue (Zhang et al., 2002), and represents approximately $50 \%$ of the stiffness of intact human skin (Zhang et al., 2007). These data indicate that PRFM is more than 600 times stiffer than the stiffest fibrin clot obtained at ambient pressure and described in the literature to date.

In routine clinical practice, the improved mechanical properties of PRFM over conventional PRP translate into a biologic matrix that is easy to handle and implant in a wide variety of tissue repair applications. The increased 
elastic modulus of the PRFM confers significant pliability and drapability which, in addition to its increased tensile strength, allows the membrane to closely conform to a wide variety of irregular surgical sites and surfaces in a manner similar to split-thickness skin autografts. Moreover, the matrix can easily be sutured to securely maintain contact with the implanted site. This makes the PRFM preferable for use in a clinical setting in which PRP has to be implanted in a specific site or where released growth factors could be washed out during an operation as in arthroscopic joint repair procedures (Maniscalco et al., 2008).

Currently available forms of autologous PRP are usually fragile, unstable, and prone to rapid fibrinolysis and dissolution following implantation. This limitation not only prevents easy handling in a clinical setting but also when the PRP gel is to be used in association with isolated cells. We have shown that the mechanical properties of PRFM are robust and long lasting and that the PRFM is effective in supporting MSC, proliferation, and survival. Scanning electron microscopy and confocal fluorescence microscopy of PRFM show a strongly enhanced average fibre dimension, on the bottom side, compared to native fibrin gels described in literature (Blomback and Okada, 1982, Blomback et al., 1989; Blomback et al., 1990; Muller et al., 1984;Okada et al., 1983; Okada and Blomback, 1983; Okada et al., 1985; Collet et al., 2004; Collet et al., 2005), with a remarkable increase to large strands, bundles and cables. The effect of branching is clearly evident on this side of the membrane. At this point, the large bundles and cables appear to be compressed to the bottom of the container where the centrifugal force is maximal during PRFM preparation. For this reason, the fibres are strongly deformed compared to their natural shape. In contrast, on the upper side of the matrix the texture appears to be coarse, with a lot of twisted and bended fibres arranged in large, coiled bundles, frequently aggregated in long cables. This arrangement could contribute to the strongly enhanced mechanical properties of this fibrin-based material, specifically the elastic modulus and the elongation at break. More studies are needed to clarify the relationship between fibrinogen concentration, membrane thickness, platelet concentration and the mechanical properties of the PRFM. The algorithm linking centrifugal force applied to PRP, or to PPP, and mechanical properties of resulting fibrin constructs are worth investigation as well. We also observed that it is possible that the difference seen in fibre size across the two sides of the matrix could be due to the delay of the coagulation process. The 25 minute centrifugation at $4500 \mathrm{~g}$, at $25^{\circ} \mathrm{C}$, needed to prepare the PRFM may not be enough for fibrin synthesis and crosslinking to go to completion. This would account for the smaller fibres found on the upper surface overlaying the more densely structured fibres found on the bottom layer after high-speed centrifugation. To clarify this finding, new studies are currently underway.

The fact that the platelets are grouped together in some areas on bottom side of the membrane, instead of being evenly distributed on the membrane's entire surface, is probably due to the particular conformation of the glass at the bottom of the container. In fact, each membrane analysed shows a different distribution of platelets on bottom side of the PRFM. It seems that the platelets tend to lie on the lower level where they are pressed by the centrifugal force.

We included the in vitro studies of the quantification of growth factors released from PRFM and their ability to promote the growth of MSC to further support the "potential" of PRFM to accelerate tissue repair. Quantification of growth factor levels and the kinetics of release show similar concentrations of bFGF, VEGF, EGF and TGF- $\beta 1$ and similar release kinetics with previous published results (Mazzucco et al., 2009). This is particularly remarkable considering that the method used to obtain the conditioned media was different in that the FIBRINET $^{\circledR}$ kit has been used to produce a matrix gel (Mazzucco et al., 2009) while in our study it has been used to produce a membrane. Further studies are needed to investigate whether the matrix gel and the membrane release growth factors with a similar kinetics.

Our results further show that growth factors released from PRFM can support MSC proliferation. This is consistent with published results that show that growth factors released from platelets have the potential to stimulate MSC proliferation (Doucet et al., 2005; Lucarelli et al., 2003). This finding is relevant in that it supports a therapeutic role of the PRFM alone if implanted in a site in which local MSC could be recruited to heal the damaged tissue. However, further studies will be needed to clarify whether MSC grown in PRFM keep the characteristics and phenotype of MSC such as multi-lineage differentiation pluripotency and maintenance of MSC markers. In vivo experiments employing PRFM itself or together with MSC will be needed to confirm the conclusion that PRFM accelerates tissue repair by itself or associated with cells.

In conclusion, production of a dense, cross-linked, physically robust PRFM made of intact platelets and fibrin by high-speed centrifugation in the absence of exogenous thrombin, yields an ideal scaffold for use in tissue repair by itself (O'Connell et al., 2006; O'Connell et al., 2008; Maniscalco et al., 2008) or in conjunction with exogenous viable cells. PRFM produced by the FIBRINET $^{\circledR}$ system is so different in makeup and physical characteristics as to be significantly distinct and separate from conventional platelet-rich plasmas. PRFM can be easily tailored to the desired shape by altering the shape of the second container. In these studies we employed a glass bottle as second container to produce a disk-shaped membrane. It is interesting to note that with this shape, speed and duration of centrifugation, most of the platelets concentrate in one side of the membrane. This sidedness may have clinical implications for enhanced tissue repair. It would also apply to isolated cell preparations, such as MSC or other cell types added to the PRFM. Further studies using a larger number of donors are currently underway to confirm the viability, growth factor release and biological response of the platelets within and released from PRFM. 


\section{Acknowledgements}

The authors are grateful to Ms. P. Dimopoulou and Cristina Ghinelli for editorial assistance and to Dr. Sergio Lodi and Prof. Pietro Maniscalco for their invaluable assistance and advice during the preparation of this paper. We are grateful to Cascade Medical Enterprises, LLC, for donating the FIBRINET $^{\circledR}$ kits for our study and for support for the mechanical testing and the confocal microscopy.

\section{Conflict of Interest}

Dr. S. O'Connell is a shareholder and receives monetary compensation as a consultant of Cascade Medical Enterprises. Dr. R. Beretta is a shareholder of Cascade Medical Enterprises.

\section{References}

Azzena B, Mazzoleni F, Abatangelo G, Zavan B, Vindigni V (2007) Autologous platelet-rich plasma as an adipocyte in vivo delivery system: case report. Aesthetic Plast Surg 32: 155-161.

Bale MF, Muller MF, Ferry JD (1985) Rheological studies of creep and creep recovery of unligated fibrin clots: comparison of clots prepared with thrombin and ancrod. Biopolymers 24: 461-482.

Blomback B, Okada M (1982) Fibrin gel structure and clotting time. Thromb Res 25: 51-70.

Blomback B, Carlsson K, Hessel B, Liljborg A, Procyk R, Aslund N (1989) Native fibrin gel networks observed by $3 \mathrm{D}$ microscopy, permeation and turbidity. Biochim Biophys Acta 997: 96-110.

Carr ME, Carr SL, Merten SR (1995) Effects on ionic and non-ionic contrast media on clot structure, platelet function and thrombolysis mediated by tissue plasminogen activator plasma clots. Haemostasis 25: 172-181.

Cervelli V, Gentile P, Grimaldi M (2009a) Regenerative surgery: Use of fat grafting combined with platelet-rich plasma for chronic lower- extremity ulcers. Aesthetic Plast Surg 33: 340-345.

Cervelli V, Gentile P, Grimaldi M (2009b) Use of cell fat mixed with platelet gel in progressive hemifacial atrophy. Aesthetic Plast Surg 33: 22-27.

Cervelli V, Gentile P, Scioli MG, Grimaldi M, Spagnoli LG, Orlandi A (2009c) Application of platelet-rich plasma to fat grafting during plastic surgical procedures: Clinical and in vitro evaluation. Tissue Eng Part C Methods 15: 625-634

Cervelli V, Palla L, Pascali M, De Angelis B, Curcio BC, Gentile P (2009d) Autologous platelet-rich plasma mixed with purified fat graft in aesthetic plastic surgery. Aesthetic Plast Surg 33: 716-721.

Chung YL, Shu C (1990) Fibrinogen, thrombosis, coagulation and fibrinolysis. In: Native Fibrin Gel Networks and Factors Influencing Their Formation in Health and Disease (Blomback B, Carlsson K, Banerjee D, Hamsten A, Hessel B, Procyk R, Silveira A, Zacharski L, eds.). Plenum Press New York, London, pp. 1-23.
Collet JP, Nagaswami C, Farrell DH, Montalescot G, Weisel JW (2004) Influence of fibrinogen splice variant on fibrin physical properties and fibrinolysis rate. Arterioscler Thromb Vasc Biol 24: 382-386.

Collet JP, Moen JL, Veklich YI, Gorkun OV, Lord SL, Montalescot G, Weisel JW (2005) The aC domains of fibrinogen affect the structure of the fibrin clot, its mechanical properties, and its susceptibility to fibrinolysis. Blood 106: 3824-3830.

Cummings CL, Gawlitta D, Nerem RM, Stegemann JP (2004) Properties of engineered vascular constructs made from collagen, fibrin, and collagen-fibrin mixtures. Biomaterials 25: 3699-3706.

Doucet C, Ernou I, Zhang Y, Llense JR, Begot L, Holy X, Lataillade JJ (2005) Platelet lysates promote mesenchymal stem cell expansion: a safety substitute for animal serum in cell-based therapy applications. J Cell Physiol 205: 228-236.

Fennis JP, Stoelinga PJ, Jansen JA (2004) Mandibular reconstruction: a histological and histomorphometric study on the use of autogenous scaffolds, particulate corticocancellous bone grafts and platelet rich plasma in goats. Int J Oral Maxillofac Surg 33: 48-55.

Franchini M, Rocca G, Bosinelli A, Governa M, Olzer D, Fiorini S, Aloisi O, Scalvi A, Gandini G, Barisoni D, Marcer M, Aprili G (2006) Use of platelet gel and bone from tissue bank in regenerative medicine as a multidisciplinary model. Blood Transfus 4: 296-310.

Fulton JE (2003) Breast contouring with "gelled" autologous fat: a 10-year update. Intl J Cosm Surg Aes Dermatol 5: 155-163.

Gamradt SC, Rodeo SA, Warren RF (2007) Platelet rich plasma in rotator cuff repair. Techniques in Orthopaedics 22: 26-33.

Hibi H, Yamada Y, Kagami H, Ueda M (2006) Distraction osteogenesis assisted by tissue engineering in an irradiated mandible: a case report. Int J Oral Maxillofac Implants 21: 141-147.

Ito K, Yamada Y, Naiki T, Ueda M (2006) Simultaneous implant placement and bone regeneration around dental implants using tissue-engineered bone with fibrin glue, mesenchymal stem cells and platelet-rich plasma. Clin Oral Implants Res 17: 579-586.

Kitoh H, Kitakoji T, Tsuchiya H, Mitsuyama H, Nakamura H, Katoh M, Ishiguro N (2004) Transplantation of marrow-derived mesenchymal stem cells and plateletrich plasma during distraction osteogenesisa preliminary result of three cases. Bone 35: 892-898.

Kitoh H, Kitakoji T, Tsuchiya H, Katoh M, Ishiguro N (2007) Distraction osteogenesis of the lower extremity in patients with achondroplasia/hypochondroplasia treated with transplantation of culture-expanded bone marrow cells and platelet-rich plasma. J Pediatr Orthop 27: 629634.

Leitner GC, Gruber R, Neumüller J, Wagner A, Kloimstein P, Höcker P, Körmöczi G, Buchta C (2006) Platelet content and growth factor release in platelet-richplasma: a comparison of four different systems. Vox Sang 91: $135-139$.

Lucarelli E, Beccheroni A, Donati D, Sangiorgi L, Cenacchi A, Del Vento AM, Meotti C, Zambon Bertoja A, 
Giardino R, Fornasari PM, Mercuri M, Picci P (2003) Platelet-derived growth factors enhance proliferation of human stromal stem cells. Biomaterials 24: 3095-3100.

Man D, Plosker H, Winland-Brown JE, Saltz R (2001) The use of autologous platelet-rich plasma (platelet gel) and autologous platelet-poor plasma (fibrin glue) in cosmetic surgery. Plast Reconstr Surg 107: 238-239.

Maniscalco P, Gambera D, Lunati A, Vox G, Fossombroni V, Beretta R, Crainz E (2008) The "Cascade" membrane: a new PRP device for tendon ruptures. Description and case report on rotator cuff tendon. Acta Biomed 79: 223-226.

Marx RE, Carlson ER, Eichstaedt RM, Schimmele SR, Strauss JE, Georgeff KR (1998) Platelet-rich plasma: Growth factor enhancement for bone grafts. Oral Surg Oral Med Oral Pathol Oral Radiol Endod 85: 638-646.

Mazzucco L, Medici D, Serra M, Panizza R, Rivara G, Orecchia S, Libener R, Cattana E, Levis A, Betta PG, Borzini $\mathrm{P}$ (2004) The use of autologous platelet gel to treat difficult-to-heal wounds: a pilot study. Transfusion 44: 1013-1018.

Mazzucco L, Balbo V, Cattana E, Borzini P (2008) Platelet-rich Plasma and Platelet gel preparation Plateltex ${ }^{\circledR}$. Vox Sang 94: 202-208.

Mazzucco L, Balbo V, Cattana E, Guaschino R, Borzini P (2009) Not every PRP is born equal. Evaluation of growth factor availability for tissue through our PRP-gel preparations: FIBRINET $\AA$, RegenPRP-kit $\AA$, Plateltex $\AA$, and one manual procedure. Vox Sang 97: 110-118.

Muller FM, Ris H, Ferry JD (1984) Electron microscopy of fine fibrin clots and fine and coarse fibrin films. J Mol Biol 174: 369-384.

O’Connell SM, Impeduglia T, Hessler K, Wang X, Carroll RJ, Dardik H (2006) Autologous platelet-rich fibrin matrix as a stimulator of healing of chronic lower extremity ulcers. Wound Rep Regen 14: A76.

O’Connell SM, Impeduglia T, Hessler K, Wang XJ, Carroll RJ, Dardik H (2008) Autologous platelet-rich fibrin matrix as cell therapy in the healing of chronic lowerextremity ulcers. Wound Rep Regen 16: 749-755

Okada M, Blomback B (1983) Calcium and fibrin gel structure. Thromb Res 29: 269-280.

Okada M, Blomback B, Block M (1983) Effect of albumin and dextran on fibrin gel structure. Thromb Haemost 50: 201 (Abstr 613).

Okada M, Blomback B, Chang MD, Horowitz B (1985) Fibronectin and fibrin gel structure. J Biol Chem 260: 18111820 .

Oliver MH, Harrison NK, Bishop JE, Cole PJ, Laurent GJ (1989) A rapid and convenient assay for counting cells cultured in microwell plates: application for assessment of growth factors. J Cell Sci 92: 513-518.

Pieri F, Lucarelli E, Corinaldesi G, Iezzi G, Piattelli A, Giardino R, Bassi M, Donati D, Marchetti C (2008) Mesenchymal stem cells and platelet-rich plasma enhance bone formation in sinus grafting: a histomorphometric study in minipigs. J Clin Periodontol 35: 539-546.

Sánchez M, Anitua E, Azofra J, Andía I, Padilla S, Mujika I (2007) Comparison of surgically repaired Achilles tendon tears using platelet-rich fibrin matrices. Am J Sports Med 35: 245-251.
Simon BI, Zatcoff AL, Kong JJW, O’Connell SM (2009) Clinical and histological comparison of extraction socket healing following the use of Autologous plateletrich fibrin matrix (PRFM) to ridge preservation procedures employing demineralized freeze dried bone allograft material and membrane. Open Dent J 3: 92-99.

Urech L, Bittermann AG, Hubbell JA, Hall H (2005) Mechanical properties, proteolytic degradability and biological modifications affect angiogenic process extension into native and modified fibrin matrices in vitro. Biomaterials 26: 1369-1379.

Virchenko O, Aspenberg P (2006) How can one platelet injection after tendon injury lead to a stronger tendon after 4 weeks? Interplay between early regeneration and mechanical stimulation. Acta Orthop 77: 806-812.

Weisel JW (2004) The mechanical properties of fibrin for basic scientists and clinicians. Biophys Chem 112: $267-$ 276.

Zhang D, Eggleton CD, Arola DD (2002) Evaluating the mechanical behaviour of arterial tissue using digital image correlation. Exp Mech 42: 409-416.

Zhang GA, Ning FG, Zhao NM (2007) Biomechanical properties of four dermal substitutes. Chinese Med J 120: 1454-1455.

\section{Discussion with Reviewers}

Reviewer I: Given the interesting mechanical properties and their release of growth factors (PDGF, VEGF, EGF, BMP-2 especially), what do you expect from the engraftment and in vivo performance of PRFM, alone or loaded with cells? Which clinical applications would be the most obvious for such a material?

Authors: The growth factors released by platelets in response to stimulation (EGF, PDGF-AA and BB, IGF-I and II, bFGF, VEGF, TGF- $\beta 1$ and 2 , etc) are the same as those involved in the initial phases of repair of most, if not all tissues of the body. Providing them in a concentrated biologic form, in their normal ratios (as opposed to excessive concentrations of a single growth factor) and capable of gradual release to the repair site over days and weeks would serve to augment healing and repair in a wide variety of clinical applications. This would be particularly true in cases where healing is compromised (i.e., poor perfusion/ischemia, repeated trauma, large/critical-size defects, underlying systemic pathology, etc.). As to engraftment, clinical experience in the treatment of lower extremity ulcers indicates that the membrane persists for 1 to 2 weeks before being absorbed in the healing wound bed (O'Connell et al., 2008). This is a fibrin matrix with intact platelets as opposed to a collagen matrix with living, proliferatively competent cells such as an autograft. As such the PRFM could be considered more of a cell therapy than a viable graft capable of 'take'. Clinical applications that can benefit from the PRFM therapy are many. In addition to difficult to heal leg ulcers, other potential applications include orthopaedic applications such as rotator-cuff repair, ligament repair (ACL, PCL, etc.), lumbar spine fusion, cranial maxillofacial reconstructive surgery (large sinus lifts, alveolar defect reconstruction, 
prognathic maxillar and mandibular reconstruction), pressure ulcers, long bone non-union, etc. In short, the PRFM membrane could be of great potential benefit in both soft tissue and bony repair where surface are coverage and augmented healing is critical. Concerning the question, when it is appropriate to add stem cells or other cells to PRFM, much more clinical work needs to be done. However, one obvious type of application would be in those situations where there are insufficient viable cells and tissue at or near the repair site for the PRFM to stimulate. Examples of such applications could include full thickness wounds such as large third degree burns, traumatic wounds or ulcers that extend to exposed tendon and bone. This would also be expected with critical-volume bone defects where scaffold materials (demineralized bone matrix, tri-calcium phosphate/hydroxy-apatite, etc.) and stem cells would be useful additions to the PRFM.

Reviewer I: The authors describe a major heterogeneity of the fibrin network throughout the material. What could be the advantages and disadvantages of this heterogeneity in therapeutic applications?

Authors: We maintain that the exceptional mechanical characteristics of the PRFM are directly related to the ultrastructure of each membrane. The presence of large twisted bundles and cables, mixed with bent fibres and fused fibrin structures, is the basis of this extraordinary elastic modulus and as such, is close to that of a thin latex membrane. Concerning inter-patient variation, several years of clinical development experience using the PRFM has demonstrated minimal inter-patient variability with respect to mechanical properties and size of the membrane construct. The experience of a number of independent clinical investigators has been that gels and membranes made from this autologous fibrin material are very reliable for use in a variety of different surgical applications. In general, we found the frequency of membranes with poor mechanical properties to be less than 1 in 200. The most frequent cause of membranes with poor characteristics was concomitant drug therapy that interfered with the coagulation process, for example patients on maintenance aspirin, Plavix, low-molecular weight heparin or other anticoagulant therapy. This is an area of continual interest and surveillance to determine the extent and cause of interpatient variability in the production and clinical application of PRFM.

Reviewer II: One of the interesting properties of the PRP for tissue engineering (added to the growth factors release etc) is that it can be mixed with cells of interest and injected before jellification. Obviously, this cannot be the case with Fibrinet ${ }^{\circledR}$, so it could only be used through invasive procedure. In addition, only materials of small size could be produced, I guess. I would be interested in having your opinion on the foreseen clinical applications.

Authors: One of the distinctive characteristics of the Membrane kit is, that it has enhanced mechanical properties compared to PRP products obtained with commercially available devices or manual procedures; therefore, it does not produce an injectable material. However, the final product has dimensions that are compatible with mini-invasive and arthroscopic orthopaedic surgical procedures, where it is delivered and anchored directly to the defect. A project is being carried out to manufacture a plastic disposable cartridge to produce a square-shaped PRFM of considerably larger size at the point of care. For example: from $20 \mathrm{~mL}$ of PRP we can get a $5 \times 5 \mathrm{~cm}$ square membrane. In another project we planned to produce a large size membrane to be stored in the refrigerator for many months, in sterile packages, to be used when needed. This would be produced with the help of a specially-designed axial centrifuge. 